SIMPÓSIO: Tópicos fundamentais para a formação e o desenvolvimento docente para professores dos cursos da área da saúde Capítulo XII

\title{
Avaliação de programas educacio- nais nas profissões da saúde: conceitos básicos
}

\section{Program evaluation on health professions education: basic concepts}

\author{
Valdes Roberto Bollela ${ }^{1}$, Margaret Castro ${ }^{1}$
}

\begin{abstract}
RESUMO:
O conceito de avaliação de programas educacionais ainda é relativamente desconhecido de muitos professores e gestores do ensino superior e, muitas vezes, é confundido com avaliação de estudantes. Avaliação é uma das áreas que mais cresceu em importância nas últimas décadas; em parte pela pressão da sociedade e também pela necessidade de mecanismos estruturados e regulares de avaliação formal para o credenciamento e recredenciamento de cursos superiores na área da saúde. Este movimento no Brasil segue uma tendência mundial de busca de sistemas comuns de certificação e acreditação. O objetivo é garantir, tanto quanto possível, altos padrões de qualidade para programas que formam profissionais da saúde. Este artigo apresenta e discute conceitos básicos e modelos de avaliação de programas educacionais, que subsidiarão qualquer profissional da saúde/educação para compreender a essência desse tema tão amplo e desafiador.
\end{abstract}

Palavras-chave: avaliação educacional; currículo; educação médica; escolas para profissionais da saúde; garantia da qualidade dos cuidados em saúde; segurança do paciente.

\section{O que é avaliação de um programa educacional?}

Entende-se avaliação de programas na área da saúde como "uma abordagem sistemática de coleta, análise e interpretação de qualquer aspecto de um programa educacional, desde a sua concepção, de- senho, implementação elou relevância para a sociedade". Outra forma de compreensão deste conceito é de um processo de obtenção de informações sobre um programa educacional para posterior julgamento e tomada de decisão. ${ }^{1}$

O termo programa educacional pode significar o curso como um todo (ex: medicina, enfermagem,

1. Docente do Departamento de Clínica Médica da Faculdade de Medicina de Ribeirão Preto da Universidade de São Paulo (FMRP-USP).

Correspondência Departamento de Clínica Médica da FMRP-USP. Hospital das Clínicas da FMRP-USP. Avenida Bandeirantes 3900. Monte Alegre. CEP: 14049-900 - Ribeirao Preto -SP.

Artigo recebido em 22/05/2014 Aprovado para publicação em 19/06/2014 
nutrição, fisioterapia), um módulo educacional dentro do próprio curso, uma disciplina ou conjunto de disciplinas de uma mesma área, um estágio ou conjunto de estágios com características comuns (ex: internato médico). Dentro desta concepção é possível avaliar, desde práticas educacionais de um único professor até o currículo de um curso como um todo. Nas últimas décadas, cresceu significativamente a importância atribuída à avaliação de programas educacionais, devido ao crescimento do número de instituições que formam profissionais da saúde e, consequentemente, um número de egressos muito maior. Isso tem significado maiores investimentos e mais atenção tem sido dada à eficiência destes programas. Outra razão é a responsabilidade que a instituição formadora tem frente às necessidades da sociedade, o que aumenta muito o empenho dos interessados em conhecer/demonstrar a qualidade dos programas educacionais na área da saúde ${ }^{2}$. Para formar profissionais da saúde capazes de oferecer atenção à saúde de alta qualidade é essencial que o treinamento recebido durante a graduação e pós-graduação também tenha sido de alta qualidade.

\section{Diferentes opiniões a respeito do que é "qualidade"}

Medir qualidade não é simples no contexto educacional, pois existem múltiplos entendimentos sobre seu significado, desde que é avaliador dependente. Os critérios usados por cada interessado na avaliação (stakeholder) tendem a ser diferentes e isso pode influenciar em um processo avaliativo que pretende ser válido e significativo ${ }^{3}$. Professores podem ter visões distintas dos estudantes sobre a qualidade da experiência educacional, resultando em conclusões nem sempre convergentes. Para minimizar esta questão é fundamental que todos os interessados sejam comunicados e envolvidos no processo de avaliação. Outras perspectivas que precisam ser levadas em consideração na questão da qualidade são: os aspectos de custo-efetividade, checagem da adequação da avaliação ao propósito do curso, e a capacidade do programa de induzir transformação da realidade.

O conceito de avaliação de qualidade evoluiu muito ao longo das últimas décadas. Os modelos iniciais estavam mais focados em identificar, corrigir ou eliminar práticas que não alcançavam os padrões desejados ${ }^{4}$. Diferente do movimento atual, que busca desenvolver uma "cultura de qualidade" na institui- ção, visando a qualificação das práticas profissionais através do compartilhamento de valores, crenças e compromissos. Neste novo pensamento, a ênfase é na melhoria contínua e não simplesmente no controle e/ou correção das falhas. A "cultura da qualidade" na educação superior das profissões da saúde ainda é muito incipiente, mas vem crescendo e, em um tempo não muito distante, será fator indutor primordial dos processos de melhoria constante da formação dos profissionais de saúde que servirão à sociedade. O movimento crescente de responsabilização do órgão formador e dos profissionais da saúde emerge como demanda da sociedade, e deve obrigar as instituições de ensino a demonstrarem que formam profissionais qualificados e dentro das expectativas e necessidades do sistema de saúde.

\section{Avaliação de programa interna versus avaliação externa}

É importante diferenciar avaliação interna de avaliação externa. As ações de iniciativa da própria escola visando avaliar, rever e melhorar regularmente seus programas educacionais são classificadas como avaliação interna. Avaliações externas costumam ser uma opção da própria instituição que convida/contrata especialistas externos para ter acesso às informações, documentos, profissionais, com ou sem visitas in loco, e que devem emitir parecer e recomendações para tomada de decisão dos gestores dos cursos ${ }^{3}$. Os pareceres podem recomendar manutenção e ampliação de algumas práticas ou sua revisão e correção de rumos. Esta modalidade de avaliação externa ocorre em instituições que vêem o processo avaliativo como valor a ser cultivado. Existe um investimento para contar com especialistas que contribuem em processos regulares de avaliação já existentes na organização. Outra modalidade de avaliação externa, geralmente compulsória, é aquela realizada por instâncias reguladoras oficiais através de processos de credenciamento, certificação e/ou acreditação. Como exemplo podemos citar avaliações realizadas pelo Ministério da Educação do Brasil através do Conselho Nacional de Residência Médica, ou o próprio Sistema Nacional de Avaliação do Ensino Superior (SINAES). De um modo geral, toda avaliação externa, seja compulsória ou opcional, é precedida por um processo de autoavaliação (avaliação interna) que serve de base para elaboração de relatórios que são disponibilizados para os avaliadores externos. 
O SINAES é uma avaliação obrigatória para todas as instituições de ensino superior federais e privadas do Brasil, que tem um componente de avaliação interna, levado a cabo pelas comissões próprias de avaliação (CPAs) das instituições e que, periodicamente, são checadas por visitadores externos indicados pelo MEC. Estes especialistas têm a missão de avaliar a universidade (avaliação institucional) e os seus cursos (avaliação de cursos) ${ }^{5}$ Além destes componentes, o SINAES conta também com o exame nacional de desempenho dos estudantes (ENADE), que realiza provas de conhecimento direcionadas a estudantes ingressantes e aqueles que estão concluindo o curso. Todos estes elementos são levados em consideração para o credenciamento/recredenciamento dos cursos de graduação do ensino superior no Brasil.

\section{Comunicação aos interessados}

Independente do propósito ou do modelo utilizado para a avaliação de programas educacionais é fundamental que toda a informação produzida na avaliação seja comunicada aos interessados (professo- res, estudantes, gestores educacionais, gestores dos serviços de saúde parceiros, população), bem como as decisões resultantes do processo avaliativo que visam a melhoria dos processos educacionais no curso. Se isso não for feito de maneira apropriada, todo o esforço, investimento de tempo e recursos terão sido em vão.

\section{Propósitos da avaliação de programas}

Existem várias razões para uma instituição de ensino em saúde preocupar-se com a qualidade do profissional que forma. A seguir apresentamos algumas destas razões tendo como referencial o currículo do curso e seus componentes principais: necessidades dos estudantes e da sociedade, estratégias e cenários de ensino/aprendizagem, avaliação do estudante e a própria avaliação do programa educacional. $\mathrm{Na}$ tabela 1, listamos algumas das razões para que os gestores do currículo preocupem-se e implementem processos avaliativos regulares com foco na melhoria contínua do curso.

Tabela 1: Propósitos para se avaliar programas educacionais.

\begin{tabular}{|c|c|}
\hline Componente & Propósito da avaliação \\
\hline \multirow[t]{4}{*}{ Gestão do Currículo } & - Desenvolver o curricular \\
\hline & - Adequar práticas educacionais ao propósito do currículo \\
\hline & - Garantir que o programa leve aos resultados esperados (outcomes) \\
\hline & - Criar a cultura de avaliação destacando os valores da organização \\
\hline \multirow[t]{5}{*}{ Ensino e Aprendizagem } & - Atender às necessidades dos estudantes \\
\hline & - Identificar professores que precisam de capacitação pedagógica \\
\hline & - Feedback aos professores e à escola \\
\hline & - criar oportunidades para desenvolvimento docente \\
\hline & - servir de referencial para promoção na carreira \\
\hline \multirow[t]{3}{*}{ Avaliação do estudante } & - conhecer a qualidade das práticas de avaliação do estudante \\
\hline & - checar se o sistema de avaliação está adequado ao propósito do curso \\
\hline & $\begin{array}{l}\text { - Certificar a qualidade e proficiência dos estudantes nos diferentes níveis de sua } \\
\text { formação }\end{array}$ \\
\hline \multirow[t]{2}{*}{ Sociedade } & - abrir espaço para participação da sociedade \\
\hline & - cuidar que suas demandas estejam contempladas no currículo \\
\hline
\end{tabular}




\section{Modelos conceituais de avaliação de programa}

Os modelos conceituais de avaliação podem ampliar nossa compreensão sobre avaliação de um programa educacional. Serão citados, a seguir, os cinco modelos mais utilizados na área educacional e que são muitos úteis para estruturar o processo de avaliação, bem como para explicá-lo aos interessados.

\section{Ciclo de avaliação}

O ciclo de avaliação considera as diferentes fases do próprio programa, desde o planejamento e preparo da atividade educacional, seguido das atividades de ensino e aprendizagem, propriamente ditas, a coleta de dados sobre estes processos e, finalmente, a análise e reflexão com o intuito de revisão e melhoria do processo. ${ }^{6}$ Este modelo contempla sete etapas que vão desde a escolha do que será avaliado, passando pelas fontes e métodos de obtenção de dados, análise, discussão, tomada de decisão e ação. Em todas as etapas devem se considerar a comunicação com os interessados e o feedback ao término do processo que resultem em mudanças nas práticas existentes (Figura 1).

\section{Avaliação orientada por tarefas}

Outro modelo bastante utilizado para planejar e conduzir avaliação de programas é aquele orientado por tarefas e também apresenta cinco etapas. É uma proposta guiada por questões orientadoras que precisam ser respondidas durante o processo avaliativo, sendo esse modelo bastante simples e praticamente auto-explicativo (Tabela 2$)^{7}$

\section{Modelo de Kirkpatrick}

O modelo mais conhecido e utilizado na educação médica é o modelo de Kirkpatrick, descrito em 1967, e que foi proposto inicialmente para avaliar os resultados do treinamento oferecido por equipes de recursos humanos. ${ }^{8}$ A figura 2 resume esse modelo de avaliação de programas, que descreve uma série de estágios a serem avaliados e requerem estratégias e instrumentos distintos, aumentando em complexidade sua realização, interpretação e ação. Para compreendê-lo, utilizaremos como exemplo uma escola médica que decidiu ampliar suas práticas de ensino fora do hospital escola, criando um novo estágio nas unidades de saúde da família em que a universidade mantém parceria com o município. Esta decisão

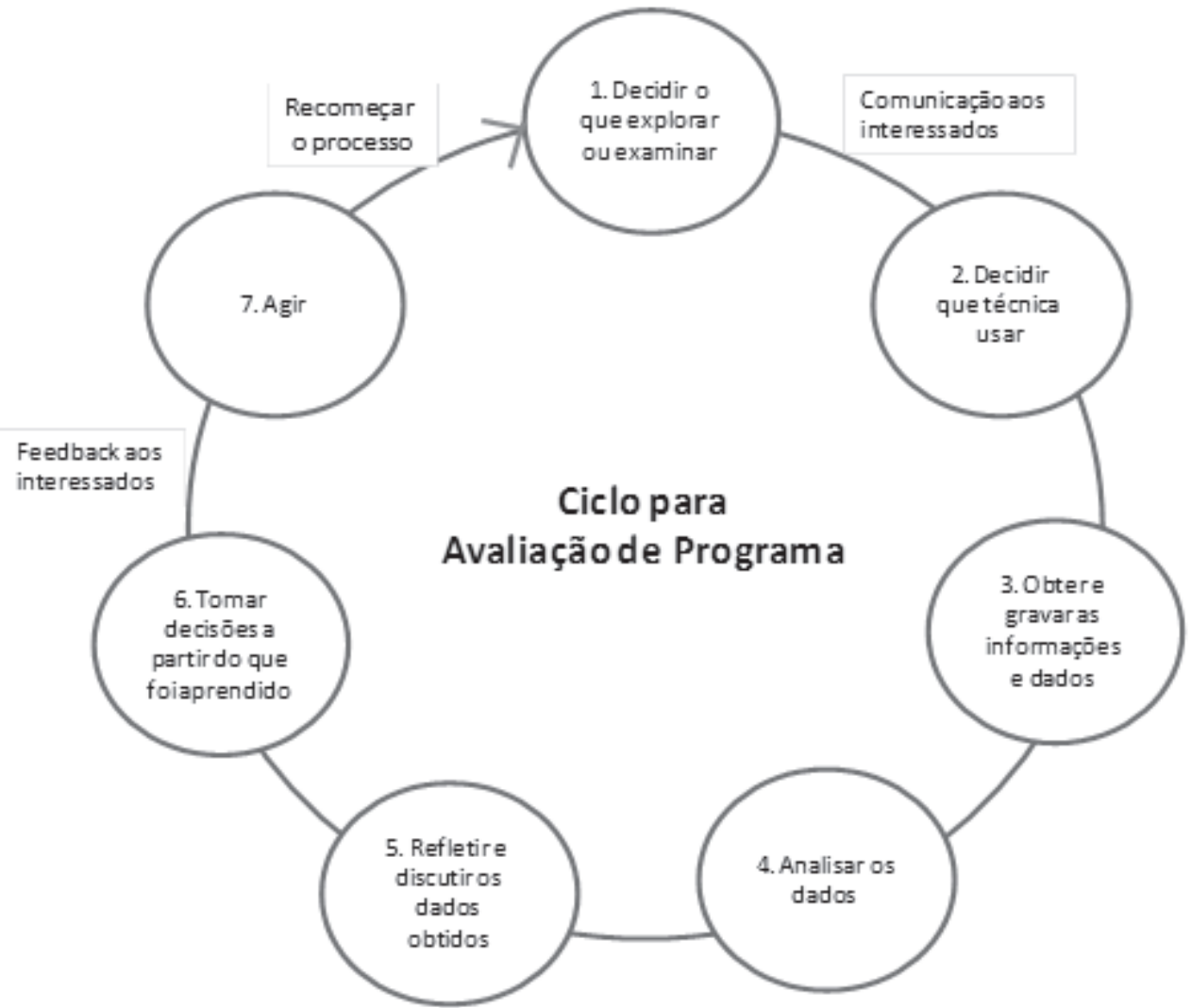

Figura 1: Ciclo de avaliação de programas. Adaptado de Edwards (1991) ${ }^{6}$ 
Tabela 2: Modelo de avaliação orientado por tarefas

\begin{tabular}{ll}
\hline Etapa & Modelo Orientado por Tarefas \\
\hline 1. Cheque a necessidade de avaliação & Questão \\
2. Determine o foco da avaliação & Por que e para quem estamos fazendo isso? \\
3. Decida sobre a metodologia & O que será avaliado? \\
4. Analise e comunique os resultados & Quem vai revisar e apresentar os resultados a todos os interessados? \\
5. Documente os resultados e ações & $\begin{array}{l}\text { Como serão documentados os resultados que servirão para a melhoria } \\
\text { do programa? }\end{array}$
\end{tabular}

foi tomada, pois, além das recomendações das diretrizes curriculares dos cursos de medicina, os gestores da escola acreditavam que este tipo de ação teria como resultado um médico com formação geral mais adequada e com mais interesse em atuar na atenção básica. A gestão do curso também acreditava que criar condições para seus estudantes e professores participarem das equipes de saúde da família do município ajudaria a escola a cumprir seu papel social junto ao sistema de saúde local. Entretanto, começaram com a implementação de apenas um estágio, pois percebiam forte resistência dentro do curso de medicina quando o assunto era mudar os cenários de prática do hospital escola para as unidades de saúde ${ }^{9}$. Segundo o modelo de Kirkpatrick, podemos avaliar o significado desta intervenção no currículo da escola analisando quatro níveis:

\subsection{Reação}

Satisfação e aprovação, que podem ser avaliadas, neste exemplo, por meio de questionários e entrevistas pela perspectiva dos estudantes, professores, gestores municipais, profissionais das unidades de saúde envolvidas que buscarão compreender as percepções de cada um após vivenciar esta experiência.

\subsection{Aprendizado}

Aquisição de conhecimento e habilidades que resultem em capacidade e/ou competência para atuar como médico na atenção básica. A complexidade deste nível é sensivelmente maior que o do anterior, bem como a qualidade e objetividade das informações. Para avaliar este nível, questionários de satisfação são insuficientes. Se quisermos saber se o estudante sabe conduzir uma consulta de pré-natal de maneira apropriada, ao término do estágio teremos de observá-lo diretamente, por exemplo através de exames que utilizem situações simuladas.

\subsection{Comportamento}

Neste nível procura-se compreender se após a intervenção e/ou treinamento, existe transferência do que foi aprendido para a prática profissional e para o ambiente de trabalho. No exemplo utilizado, esperase observar médicos egressos trabalhando na atenção básica e demonstrando proficiência nas práticas do cuidado ao paciente atendidos no serviço. Do ponto de vista institucional espera-se, a partir desta experiência piloto, uma revisão ampla do currículo de modo a confirmar e ampliar as experiências dos alunos na atenção básica, envolvendo outras especialidades que não apenas a saúde coletiva. Por exemplo, o departamento de cirurgia pode decidir organizar um novo rodízio no ambulatório da unidade de saúde, onde os estudantes realizem pequenas procedimentos ambulatoriais, sob supervisão. Este novo estágio substituiria as duas semanas que os estudantes passavam no centro cirúrgico do hospital terciário da universidade. Enfim, busca-se checar se houve mudança nos valores e práticas relacionados à educação baseada na comunidade, em um curso de medicina que mantinha práticas de ensino totalmente centradas no hospital escola.

\subsection{Resultados (impacto)}

Neste nível, as análises sempre são feitas a longo prazo. Ainda, requerem "medidas" nem sempre simples e fáceis de serem operacionalizadas. No exem- 
plo dado acima, esperaríamos que uma melhor atenção básica nas unidades de saúde da família levando a uma melhor saúde da população cuidada em decorrência da decisão anterior de se estimular a diversificação de cenários de práticas na formação médica. Não é difícil concluir que esses estudos que dão informações sobre os níveis 3 e 4 do modelo de Kirkpatrick são mais complexos e de maior custo.

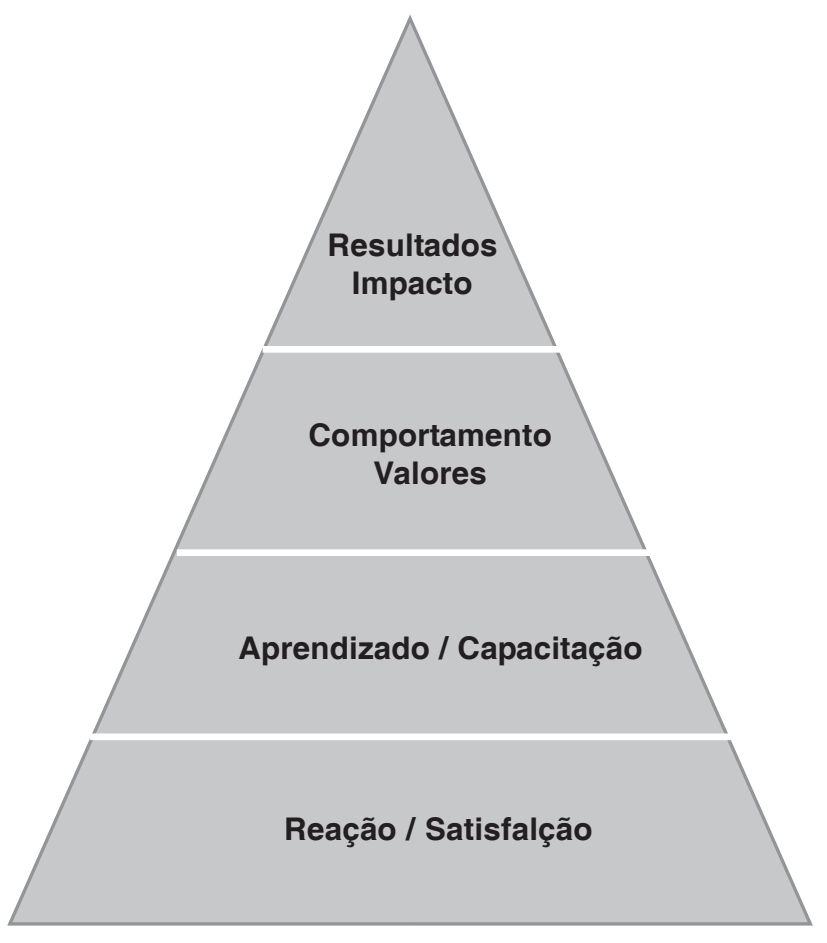

Figura 2: Modelo de Kirkpatrick para Avaliação de Programas .

\section{Modelo Lógico}

O modelo lógico é bastante usado tanto para o planejamento quanto para avaliação de programas; apresenta uma estrutura bastante racional para todas as etapas e componentes que devem ser observados durante um processo avaliativo ${ }^{10}$. É bastante linear e costuma ser criticado, pois subestima as inúmeras interrelações existentes entre pessoas interessadas e componentes existentes nos programas educacionais. Por outro lado, costuma agradar aqueles que preferem modelos simples e mais cartesianos. A tabela 3 apresenta os componentes do modelo lógico, também chamado CIPP (do inglês Context à Input à Process à Product) com as perguntas que devem ser feitas em cada etapa da avaliação.

\section{Modelos que reconhecem progra- mas educacionais como sistemas complexos}

Os modelos mais recentes de avaliação partem do princípio de que um programa educacional não é uma entidade singular, simples e estável. Muito pelo contrário, é um sistema composto por uma miríade de componentes dinâmicos que interagem em um sistema não-linear e complexo, que sofrem constantemente influências indutoras de mudança, sob regras totalmente imprevisíveis. Este conceito assume que os modelos de avaliação mais lineares seriam inadequados para programas que trazem dentro de si aspectos imponderáveis. Nesse caso, não seria adequado avaliar apenas se programa alcançou os "resultados esperados", pois muitas mudanças, para além das metas e resultados esperados, acontecem desde o planejamento até a execução de programa educacional.

Haji et al. (2013) propõem um novo modelo que difere dos descritos anteriormente por questionar a capacidade destes de lidar e compreender os complexos processos que envolvem o contexto da atenção à saúde e, em especial, da formação de profissionais para esta área ${ }^{11}$. Os autores afirmam que avaliar programas não se resume a julgamentos de mérito e/ou valor, mas que esta avaliação deve gerar informações para os gestores do currículo para que os programas educacionais possam se adaptar ao contexto e às necessidade dos estudantes e da sociedade. Espera-se que os pesquisadores desta área sejam capazes de avaliar programas de modo a gerar conhecimento para orientar o trabalho de outros educadores e dos profissionais da saúde. Neste modelo é fundamental envolver múltiplos interessados (stakeholders), combinar vários métodos para coleta e análise de dados e, finalmente, reconhecer que o processo avaliativo dura enquanto durar o programa. Enfim, é uma atividade essencial para a vitalidade do programa, bem como para que sua renovação aconteça de maneira consciente e informada. ${ }^{11}$

A figura 3 mostra a síntese dos aspectos essenciais deste modelo, que delineia sete elementos essenciais. Por este modelo, a avaliação começa na concepção do programa, reconhecendo quais os referenciais teóricos embasam as decisões tomadas. Assim, é possível esclarecer como se "espera" que a intervenção ou a nova proposta seja capaz de alcançar os objetivos esperados, que serão avaliados 
Tabela 3: Componentes e perguntas orientadoras para a utilização do modelo lógico, adaptado de Frye e Hemmer (2012) $)^{\mathbf{1 0}}$

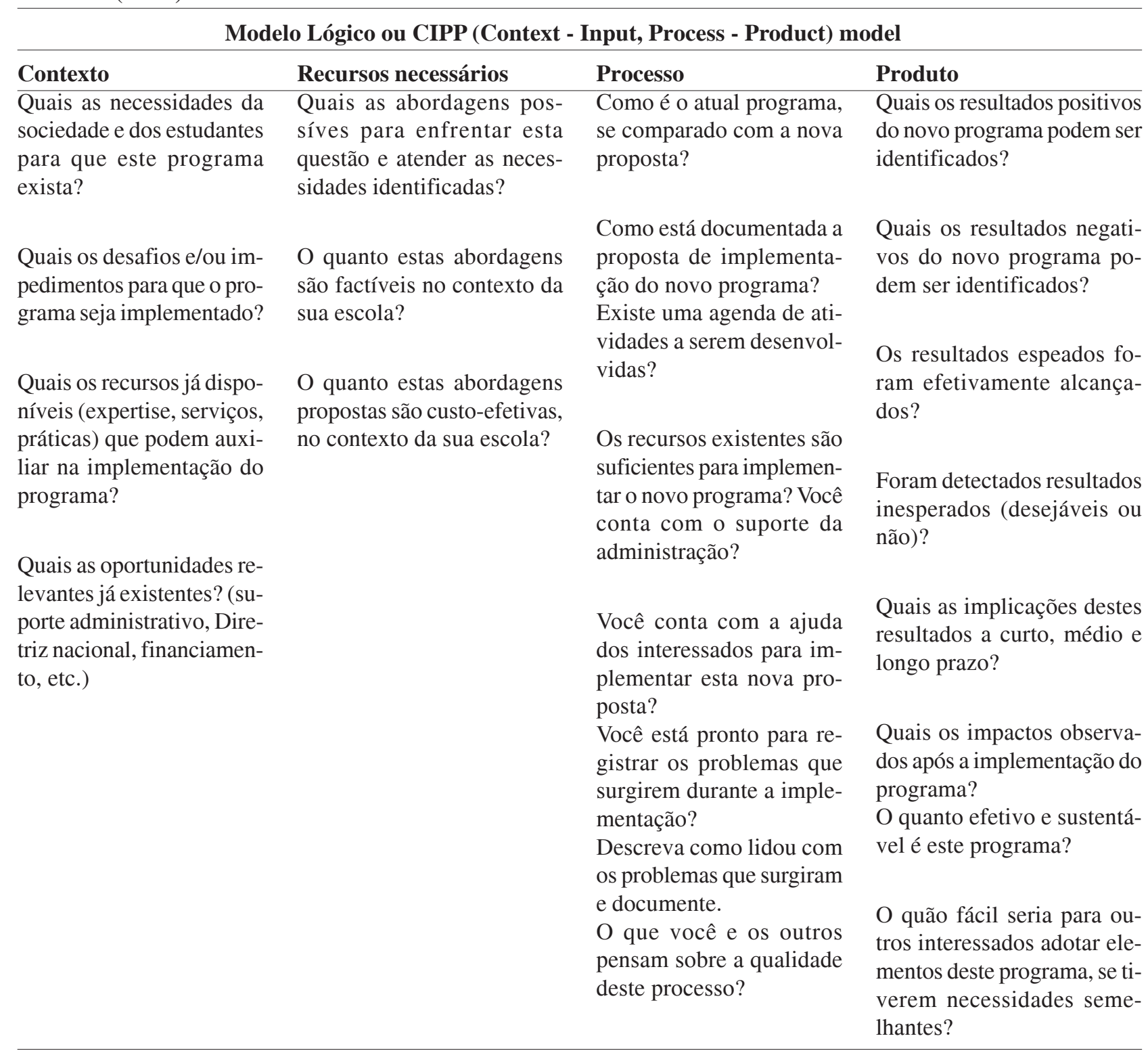

Adaptado de Frye e Hemmer (2012) ${ }^{10}$

ao final do processo de mudança. Até aqui, este modelo difere pouco dos citados anteriormente. O que ele adiciona é a necessidade de estar atento e avaliar também "o que mais aconteceu, além do esperado" durante a sua implementação. Esses aspectos são nãolineares e totalmente imponderáveis. É possível que os resultados esperados com a mudança do currículo sejam alcançados por meios que não foram planejados sendo de suma importância esse fato ser reconhecido e comunicado. Na figura 3 , existe um círculo que representa o contexto (presente em todos os outros modelos), mas neste caso, existe a crença de que o contexto será alterado pela implementação do programa, gerando mudanças no próprio contexto onde o programa está inserido. Assim, a avaliação deve considerar e checar os processos e resultados esperados, mas também deve buscar explicações adicionais, que podem emergir durante o implantação do mesmo e que podem ir muito além do que havia sido previsto e planejado pelo referencial teórico. 


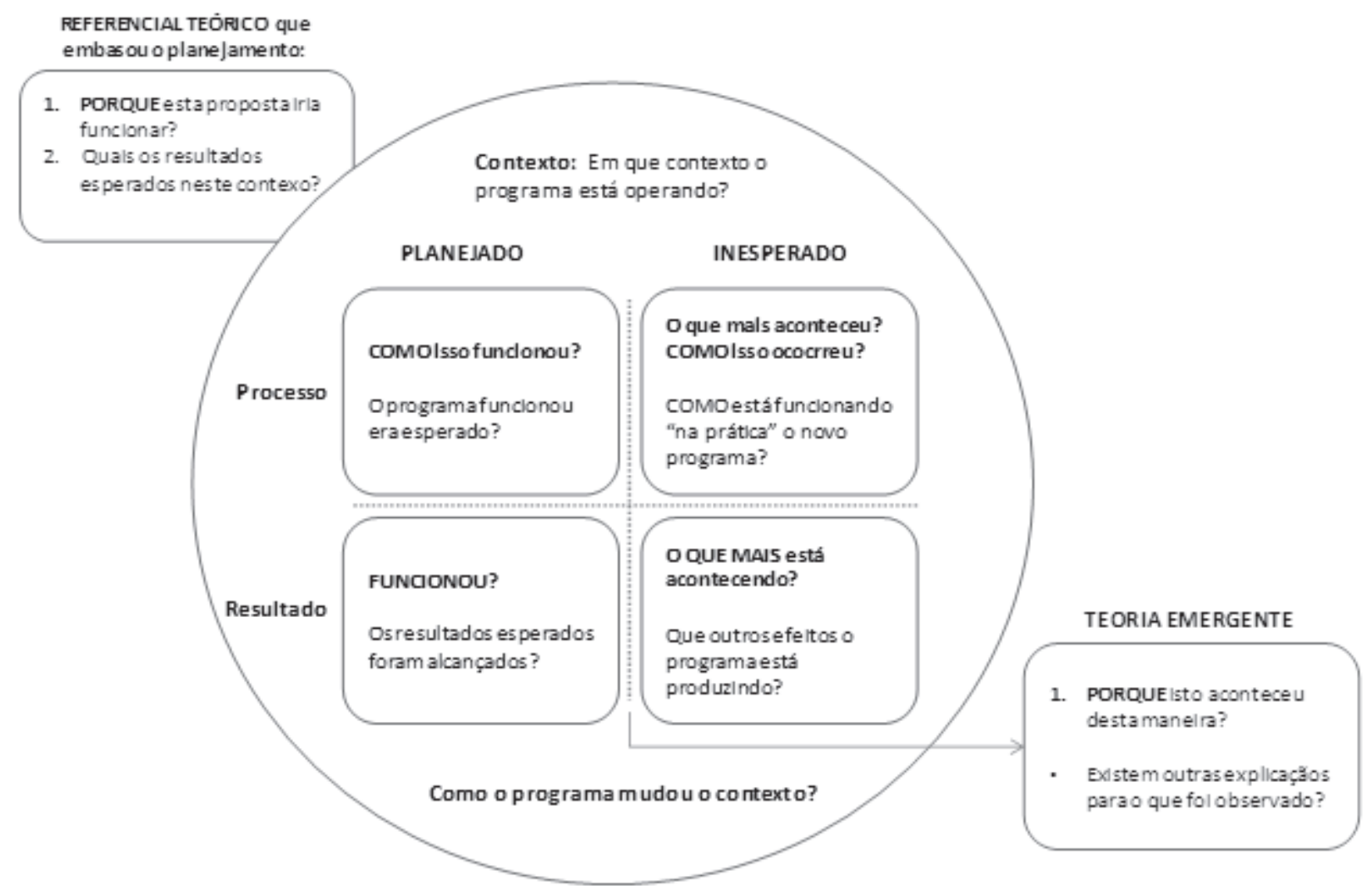

Figura 3: Modelo de avaliação de sistemas educacionais proposto dentro de um referencial teórico de alta complexidade (Haidi et al., 2013).

\section{Obtenção de dados na avaliação de programas educacionais}

Na avaliação de programas educacionais existem algumas fontes e formas de obtenção de dados para compor o conjunto de informações, que serão analisadas para gerar relatório, emissão de juízo de valor, e de tomada de decisões. A seguir citaremos as principais fontes de informação e os principais métodos de obtenção de dados para a avaliação de um programa. Maiores detalhes podem ser obtidos em referências complementares, ${ }^{12,13,14}$ desde que os métodos de obtenção e avaliação de informação não são o foco deste artigo.

\section{Fontes de informação}

Dentre as várias fontes disponíveis para obter informações relacionadas a qualidade de programas educacionais, podemos citar:

- Percepção dos estudantes: é uma fonte bem reconhecida, relevante e dominante em artigos sobre avaliação de programas nas últimas décadas. Existem muitas críticas a essa fonte, especialmente se forem as únicas disponíveis e se não forem representativas do conjunto de estudantes que vivenciaram aquela experiência ${ }^{15}$. Entretanto, não se pode imaginar a avaliação de uma experiência educacional que deixe de fora a perspectiva dos estudantes.

- Avaliação por pares: os membros do corpo docente podem avaliar-se mutuamente. Isso também é valido para o corpo técnico administrativo.

- Auto-avaliação: recurso essencial em qualquer forma de avaliação.

- Avaliação externa: membros da sociedade civil, conselhos de saúde, gestores do sistema de saúde, parceiros da escola em atividades junto à comunidade, organizações externas acreditadoras ou certificadoras.

- Egressos da escola: opinião e colocação dos mesmos no mercado de trabalho.

- Resultados dos estudantes em avaliações de desempenho, tanto internas quanto externas.

- Documentos disponíveis na instituição sobre o programa educacional.

- Evidências de boas práticas que possam ser observadas diretamente ou checadas através de entrevistas com estudantes e corpo funcional da instituição. 


\section{Métodos de obtenção das Informações}

As metodologias utilizadas na coleta de dados podem ser classificadas, de maneira geral, como métodos qualitativos, quantitativos ou mistos. $\mathrm{O}$ termo quantitativo é empregado quando a informação envolve números ou itens que podem contados e quantificados de forma objetiva. Os métodos quantitativos mais comumente usados para obter este tipo de dados são:

- Questionários

- Enquetes

- Escalas do tipo LIKERT

- Checklists

- Escalas psicométricas

- Dados demográficos

- Dados e evidências sobre a infra-estrutura para o programa

- Documentos e registros que descrevam as atividades curriculares realizadas pelos estudantes e sua duração.

- Informações obtidas dos resultados da avaliação dos estudantes, durante e/ou ao final do programa.

- Dados sobre o desempenho dos egressos em concursos ou em exames nacionais de desempenho.

O termo qualitativo envolve descrições, opiniões ou outras fontes de natureza narrativa. Os métodos clássicos de coleta de dados qualitativos incluem vários tipos de estratégia como:

- Entrevistas

- Grupos de discussão

- Observação direta

- Diários

- Auto-relatos

- Estudo de casos

- Análise de documentos como atas de comissões e instâncias colegiadas

- Registro e análise de incidentes críticos

\section{Conceito de Avaliação: “Assessment"e "Evaluation"}

$\mathrm{Na}$ literatura internacional, especialmente na Europa e Reino Unido, o termo utilizado para avaliação de programa é "evaluation", enquanto o termo "assessment" é mais utilizado para avaliar o aprendizado de estudantes e residentes. Na língua portuguesa, estes dois termos são expressos pela mesma palavra "avaliação". Deste modo, em português, quando nos referimos à avaliação no contexto educacional é fundamental que deixemos claro a que tipo de avaliação estamos nos referindo: estudantes ou programas educacionais. Cabe destacar que a avaliação do estudante (assessment) invariavelmente será um dos componentes da avaliação do programa educacional. A qualidade do egresso, medida através de estratégias reconhecidas, válidas e confiáveis será sempre um dos importantes indicadores de sucesso de um curso que forma profissionais de saúde.

\section{Avaliação de programa é o mesmo que pesquisa em educação?}

Avaliação de programa normalmente não é o melhor termo a ser empregado se o que fazemos é uma pesquisa em educação. Apesar de guardarem algumas semelhanças e exigirem os mesmos cuidados e rigores metodológicos, são processos distintos. Quando se faz pesquisa em educação, geralmente buscamse resultados generalizáveis que se aplicam em outros contextos. Outro resultado esperado em pesquisas é o da publicação dos resultados através de um processo de revisão por pares, o que requer invariavelmente a aprovação prévia de um comitê de ética para o projeto de pesquisa ${ }^{16}$. Nos processos avaliativos de um programa educacional, as informações obtidas são geralmente para uso interno e local ou, eventualmente, visam processos de certificação e/ou acreditação ${ }^{17,}{ }^{18}$. Não exigem aprovação de comitês de ética, mas devem seguir preceitos éticos no que diz respeito à confidencialidade da informação e a forma com que será divulgada internamente. Além disso, os processos avaliativos de um programa educacional devem ser conduzidos com rigor metodológico já que seus resultados orientarão a tomada de decisão visando qualificar a formação dos futuros profissionais de saúde que serão entregues à sociedade.

\section{Desafios no processo de avaliação de programas educacionais}

Para concluir esta revisão sobre este tema, destacam-se a seguir alguns cuidados que se deve ter ao planejar, implementar e/ou participar de um processo de avaliação de programa educacional. Wall (2012) descreve algumas armadilhas relacionadas ao tema que requerem atenção e devem ser evitadas ${ }^{1}$ :

- Evite só medir o que é fácil medir

- Cuidado com amostragem não significativa. A taxa de resposta a um questionário neste tipo de processo 
de avaliação deve ser de, no mínimo, 70\% dos envolvidos para garantir de que os dados são válidos.

- Evite, tanto quanto possível, métodos de obtenção de dados com baixa confiabilidade, ou seja, estratégias que não produzem resultados consistentes se forem repetidas nas mesmas condições, o que significa, em última análise, uma baixa reprodutibilidade do método.

- Fique atento às questões éticas, principalmente a confidencialidade dos participantes e interessados, no momento da divulgação dos resultados.

- Não espere que as conclusões da avaliação sejam adotadas ampla, indistinta e imediatamente. Toda instituição tem uma cultura e momentos próprios que precisam ser compreendidos e respeitados.

Para avaliar mudanças significativas na estrutura de um processo educacional é importante esperar o tempo necessário para que a nova prática possa maturar. Esse tempo é variável, mas sugerem-se pelo menos três ciclos/anos da nova prática, para que se possa concluir algo sobre os dados coletados. Nas primeiras aplicações de novas práticas pedagógicas ou de avaliação é comum que existam certa insegurança, receios e dúvidas por parte de professores e alunos.

\section{Conclusão}

Avaliação é uma atividade essencial no contexto educacional, em especial para as instituições que aceitam a responsabilidade de formar profissionais da saúde. Assim, é preciso que professores e gestores educacionais reconheçam esta prática como sendo intrínseca à prática educacional e um componente indispensável no processo de credenciamento e recredenciamento dos cursos superiores no Brasil. Mais importante é o fato de que avaliações regulares retro-alimentam a tomada de decisão que almejam melhores práticas de ensino e aprendizagem.

\section{Principais pontos de interesse}

- Considerando a responsabilidade das instituições de ensino na área da saúde é inaceitável que estas não mantenham sistemas regulares de avaliação de suas práticas pedagógicas, de avaliação dos estudantes e da qualidade do produto (profissionais da saúde) que entregam à sociedade.

- Avaliar é dar valor, só assim podemos obter informações que possibilitam adequação e melhoria contínua nos processos educacionais. Uma avaliação de qualidade deve ser rigorosa e defensável.

- Existem evidências sobre as melhores práticas na avaliação de programas educacionais que devem ser respeitadas e praticadas.

- Mantenha sempre a mente aberta. Fique atento a resultados inesperados que podem ser observados e amplificados ao término do processo avaliativo.

- Dentre as diversas razões para dar suporte aos programas de avaliação na área da saúde, destacamos a crença de que uma boa avaliação resultará sempre na melhoria da atenção à saúde da população.

\begin{abstract}
The concept of evaluation in programs of health profession education (HPE) is relatively unknown to many teachers and educational managers responsible for higher education. Often, it is misunderstood with students' assessment. The importance of evaluation in the HPE has grown in recent decades due to the pressure of society but also because structured and formal mechanisms have been required for accreditation and re-accreditation of HPE courses. The growing interest in HPE in Brazil follows a global trend of certification and accreditation programs. The objective is to ensure high quality standards for graduate health professional programs. This article presents the basic concepts and models of evaluation in HPE programs that may be used for health/educational professionals in order to better understand the essence of this broad and challenging topic.
\end{abstract}

Keywords: educational assessment; curriculum; institutional evaluation; program evaluation; medical education; quality assurance, health care; patient safety. 


\section{Referências Bibliográficas}

1. Wall D. Evaluation: improving practice, influencing policy. In: Swanwick T. Understanding Medical Education. Evidence, Theory and practice. 2010. Wiley-Blackwell. London.

2. Spencer-Mathews S. Enforce cultural change in academe. A practical case studyimplementing quality management systems I higher education. Assess Eval Higher Educ 26: 51-9, 2001.

3. Dolmans D, Stalmeijer R, vanBerkel H, Wolfhagen I. Chapter 15: Quality assurance of teaching and leraning: enhancing the quality culture. In: Dronan T, Mann K, cherpbier A, Spencer J. Medical Education. Theory and Prectice. 2011. Churchill Livingstone, Elsevier. London.

4. Sallis E. Total quality management in education, UK, 2002 , Kogan Page

5. Brasil. Ministério da Educação, Instituto Nacional de Estudos e Pesquisas Educacionais Anísio Teixeira. Sistema Nacional de Avaliação da Educação Superior: da concepção a regulamentação., 2a. ed. ampl. Brasília, 2004. Lei oㅡ 10.861, de 14 de abril de 2004, publicada no D.O.U. em 15 de abril de 2004.

6. Edwards J. Evaluation in adult and further education: a practical handbook for teachers and organisers. Liverpool: Workers' Educational Association, 1991.

7. Musick D. A conceptual model for program evaluation in graduate medical education. Acad Med. 81: 759-65.

8. Kirkpatrick D. Evaluation of training. In: Craig RL, Bittel LR, eds. Training and Development Handbook. New York, NY: McGraw-Hill 1967;87-112.
9. Bollela VR, Machado JLM. Internato baseado em competencia:. Bridging the gaps. 2010. Editora Medvance. São Paulo-Brasil.

10. Frye AW \& Hemmer PA. Program evaluation models and related theories: AMEE Guide № 67. Medical Teacher. 2012; 34: e288-e299.

11. Haji F, Morin MP, Parker K. Rethinking programme evaluation in health professions education: beyond 'did it work?'. Med Educ. 2013: 47: 342-51.

12. Hutchinson L. Evaluating and researching the effectiveness of educational interventions. BMJ 1999;318:1267-9.

13. Kuper A, Reeves S, Levinson W. An introduction to reading and appraising qualitative research. BMJ 2008;337:a288.

14. Fraenkel JR and Wallen NE. The nature of qualitative research. In: How to design and evaluate research in education. McGraw Hill Press. 2003; part (5): 427-48.

15. Berk RA. Thirteen strategies to measurescollege teaching. 2006. Stylus publishing LLC. Sterling,VA.

16. Morisson J. ABC of learning and teaching in medicine: Evaluation. BMJ. 2003; 326(7385):385-7.

17. Viswanathan HN, Salmon JW. Accrediting organizations and quality improvement. American Journal of Managed Care. 2000; 6: 1117-30.

18. Montagu D. Accreditation and other external quality assessment systems for healthcare: Review of experience and lessons learned. DFIP Health System Resource Center. 2003; p.p. 1-38. 